\title{
Shear force-based genetic screen reveals negative regulators of cell adhesion and protrusive activity
}

\author{
Thomas J. Lampert ${ }^{a}$, Nadine Kamprad ${ }^{b}$, Marc Edwards $^{a}$, Jane Borleis ${ }^{a}$, Ayende J. Watson ${ }^{a}$, Marco Tarantola ${ }^{b}$, \\ and Peter N. Devreotes ${ }^{a, 1}$ \\ ${ }^{a}$ Department of Cell Biology, Johns Hopkins University School of Medicine, Baltimore, MD 21286; and ${ }^{\mathrm{b}}$ Max Planck Institute for Dynamics and
Self-Organization, 37077 Göttingen, Germany \\ Contributed by Peter N. Devreotes, July 21, 2017 (sent for review October 6, 2016; reviewed by Adam Kuspa and Margaret A. Titus)
}

\begin{abstract}
The model organism Dictyostelium discoideum has greatly facilitated our understanding of the signal transduction and cytoskeletal pathways that govern cell motility. Cell-substrate adhesion is downstream of many migratory and chemotaxis signaling events. Dictyostelium cells lacking the tumor suppressor PTEN show strongly impaired migratory activity and adhere strongly to their substrates. We reasoned that other regulators of migration could be obtained through a screen for overly adhesive mutants. A screen of restriction enzyme-mediated integration mutagenized cells yielded numerous mutants with the desired phenotypes, and the insertion sites in 18 of the strains were mapped. These regulators of adhesion and motility mutants have increased adhesion and decreased motility. Characterization of seven strains demonstrated decreased directed migration, flatness, increased filamentous actin-based protrusions, and increased signal transduction network activity. Many of the genes share homology to human genes and demonstrate the diverse array of cellular networks that function in adhesion and migration.
\end{abstract}

cell adhesion | cell migration | chemotaxis | Dictyostelium | PTEN

$\mathbf{M}$ uch of what we know about amoeboid migration, an integral mode of cellular locomotion seen in metazoans, including cells of the immune system and metastasizing cancer cells, derives from studies of Dictyostelium discoideum. As a haploid eukaryotic cell with strong homology to metazoans, this model organism has been an essential tool for genetic analysis of these conserved cellular behaviors. During amoeboid migration, cells extend projections that, with the addition of changes in specific and nonspecific adhesions to the substratum, lead to a progressive movement of the cell (1-3). These projections are controlled by nucleators of filamentous-actin (F-actin) like Arp $2 / 3$ and actin-binding proteins, such as myosin II, that establish the internal forces needed to mobilize regions of the cell membrane leading to propulsions like pseudopods and ultimately amoeboid migration (4-7). In randomly migrating cells, this protrusive and contractile behavior of the cytoskeletal network is controlled by spontaneous activation of signal transduction networks (8). In chemical gradients, chemotaxis occurs when a cell's extracellular receptors bind specific molecules influencing intracellular signal transduction and thus alter the dynamics of the cytoskeleton in a directed fashion $(9,10)$.

Genetic analyses have revealed numerous genes that control cell migration (11-13). Although many of the screens in multicellular development and wound healing have provided insights into more complex migrations in tissues, combinatorial genetics and single cell observations have the potential of defining the genes involved in the basic mechanisms of individual cellular migration. Specifically, in Dictyostelium there have been nearly a hundred genes implicated in cell migration from both forward and reverse genetic methodologies $(9,14)$. These genes comprise the classical cytoskeletal components as one would expect in addition to many signal transduction components. One of the critical discoveries made in Dictyostelium was the role that the phosphoinositide pathway plays in the regulation of cell migration (15-17). The local accumulation of phosphatidylinositol $(3,4,5)$-triphosphate (PIP3) at the plasma membrane and the creation of activated membrane patches must be tightly regulated as it allows for the binding and activation of numerous intracellular signaling proteins (18). Deletion of phosphatase and tensin homolog (PTEN) that negativity regulates the levels of PIP3 markedly increases the activity of the cytoskeleton. This leads to strong defects in both random and directed migration associated with the resulting elevated levels of PIP3 (19).

Capitalizing on the conserved mechanisms of migration, the accessible genetics, and the phenotype of pten- cells, we used Dictyostelium to seek new regulators of migration (19, 20). Cells lacking PTEN provided an excellent control to facilitate the development of such a screen as the increased level of PIP3 on the plasma membrane and cytoskeletal activity leads to simultaneous multidirectional projections and a concomitant increased cell-substrate adhesion. Moreover, Dictyostelium pten- cells do not display the increased proliferation rates seen in mammalian cells lacking this gene, which would complicate the screen. It appears that Dictyostelium cells do not possess PIP3-dependent cell cycle checkpoints as in animal cells $(19,21,22)$. We reasoned that other negative regulators in signaling or cytoskeletal pathways would lead to a similar phenotype as seen in pten- cells consisting of increased protrusive activity, cell spreading, contact area, and substrate adhesion. Therefore, using pten- cells we designed a forward genetic screen in populations of cells carrying random insertions controlling cell migration and substrate adhesion (23). Screening more directly for such defects may reveal different sets of genes than

\section{Significance}

We report a forward genetic screen to identify genes involved in cell adhesion and motility. Cells with mutations in these genes have increased adhesion, flattened morphology, and decreased motility. The mutants display increased cytoskeletal and signal transduction network activity suggesting that these genes are negative regulators. The GFP-tagged localization of these proteins shows the remarkable diversity in the regulation of these cell behaviors. Several of the identified proteins have strong homologs throughout metazoans and have relevance to human disease. Because many of the resulting mutant phenotypes are similar to those of cells lacking PTEN or expressing active Ras GTPases, these gene families are promising cancer targets in humans. Better understanding of these pathways holds the possibility for therapeutic intervention.

Author contributions: T.J.L., N.K., M.E., J.B., A.J.W., M.T., and P.N.D. designed research; T.J.L., N.K., M.E., J.B., A.J.W., and M.T. performed research; T.J.L., N.K., M.E., J.B., and M.T. contributed new reagents/analytic tools; T.J.L., N.K., M.E., J.B., A.J.W., M.T., and P.N.D. analyzed data; and T.J.L., N.K., M.E., J.B., A.J.W., M.T., and P.N.D. wrote the paper. Reviewers: A.K., Baylor College of Medicine; and M.A.T., University of Minnesota. The authors declare no conflict of interest.

Freely available online through the PNAS open access option.

${ }^{1}$ To whom correspondence should be addressed. Email: pnd@jhmi.edu.

This article contains supporting information online at www.pnas.org/lookup/suppl/doi:10. 1073/pnas.1616600114/-/DCSupplemental. 


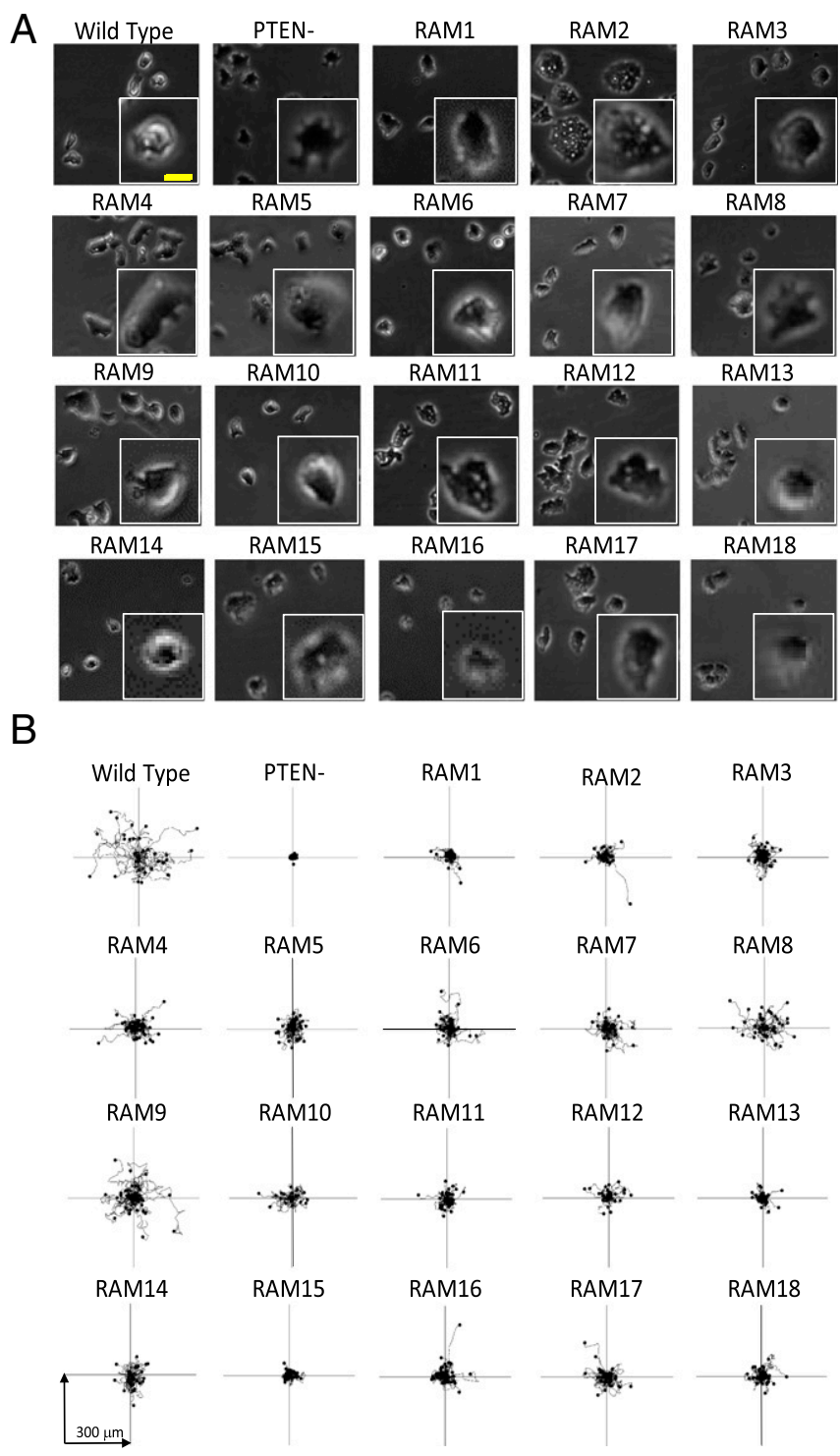

Fig. 1. Phase images and random motility profiles of vegetative RAM mutants. (A) A single frame was taken from a random migration time-lapse video to show the morphology of RAM mutants. The inset shows a closer examination of a single cell. (Yellow scale bar, $5 \mu \mathrm{m}$.) $(B)$ For one sample video, the image sequences were analyzed, and the tracks were centered at an origin to compare random migrations. $n=30-40$ cells.

those found in previous screens which have identified genes involved in the acquisition of chemotactic competence.

\section{Results}

Novel Regulators of Adhesion and Migration. Reasoning that novel genes regulating cell motility could be identified by monitoring adhesion, our screen used a shear flow assay to select for more adhesive mutants. To establish the screening procedure, we used the pten- cell line as a positive control. As shown in SI Appendix, Fig. $\mathrm{S} 1 A$, pten- cells are phase dark, suggesting they are flattened compared with wild-type cells $(19,24,25)$. We subjected monolayers of wild-type or pten- cells to a shear flow such that less than $20 \%$ of wild-type and $80 \%$ of the pten- cells remained adhered to the substrate (SI Appendix, Fig. S1B). To reconstruct the planned screen, we mixed pten- cells with an abundance of wild-type cells. The cells were subjected to cycles of shear flow selection followed by growth intervals. Phase-contrast microscopy shows that an increasing fraction of the cells in the mixed population became phase dark pten- cells over time (SI Appendix, Fig. S1C). There was little change in nonmutagenized populations of wild-type, or pten-, cells exposed to the same protocol (SI Appendix, Fig. S1D). The procedure was then tested on restriction enzyme-mediated integration (REMI) mutagenized populations to assess whether cells with modified adhesion are present. After 13 cycles of selection, the mutagenized REMI population showed a significant drift toward the phenotypes typically associated with pten- cells including increased adhesion, decreased random migration, and an increase in the number of small clonal plaques formed on a bacterial lawn (SI Appendix, Fig. S2). Clonal isolates from the now abundant overly adhesive cells line present in these populations were selected. We performed the adhesion selection screen several times on REMI mutagenized populations and are reporting on the first 18 regulators of adhesion and motility (RAM) mutants. The sites of insertion of the integrating plasmid in these mutants are included in SI Appendix, Table S1.

As shown in Fig. 1, most of the RAM mutants have a phasedark, spread morphology like pten- cells and display decreased random motility (Movies S1-S7). Table 1 shows that the increases in adhesion and decreases in migration for RAM cells relative to wild type are generally correlated. There are a few exceptions such as RAM6, which have reduced motility but not strongly increased adhesion and appear more like wild-type cells in phase microscopy (Fig. 1A) (Table 1), and RAM9, which is strongly adhesive but does not display a significant decrease in average random migration compared with wild-type cells. This was because a fraction of the RAM9 cells display a novel keratocyte-like behavior with extensive surface contact and a high, persistent, and gliding motility which skews the population's average speed (SI Appendix, Fig. S3) $(26,27)$. The remaining cells in the population have a slower motility than wild-type cells.

The RAM mutants also displayed altered chemotactic motility and progression through the developmental process. To assess whether RAM mutants differentiated appropriately we tracked the expression of the chemoattractant receptor, cAR1 (SI Appendix, Fig. S4A) (20). The RAM3 and RAM12 mutant strains show little to no expression of cAR1, the $\operatorname{sep} A$ - strain shows a

Table 1. Gene ontology, adhesion, and motility of RAM mutants

\begin{tabular}{lccc} 
Strain & Gene ontology & $\%$ of WT adhesion & $\%$ of WT speed \\
\hline pten- & Phosphatase & $363 \pm 86$ & $19 \pm 4$ \\
RAM1 & Actin binding & $231 \pm 55$ & $53 \pm 9$ \\
RAM2 & Transport ATPase & $301 \pm 71$ & $31 \pm 16$ \\
RAM3 & N/R & $237 \pm 56$ & $46 \pm 17$ \\
RAM4 & Protein binding & $265 \pm 63$ & $38 \pm 12$ \\
RAM5 & Kinase & $235 \pm 56$ & $46 \pm 4$ \\
RAM6 & N/R & $142 \pm 34^{*}$ & $43 \pm 12$ \\
RAM7 & N/R & $215 \pm 51$ & $49 \pm 14$ \\
RAM8 & Zinc/DNA binding & $229 \pm 54$ & $61 \pm 7$ \\
RAM9 & N/R & $287 \pm 68$ & $98 \pm 10^{\dagger}$ \\
RAM10 & Protein binding & $168 \pm 40$ & $76 \pm 15^{*}$ \\
RAM11 & N/R & $213 \pm 50$ & $36 \pm 1$ \\
RAM12 & Binding & $306 \pm 72$ & $32 \pm 8$ \\
RAM13 & Phosphatase & $319 \pm 76$ & $23 \pm 4$ \\
RAM14 & N/A & $165 \pm 39 *$ & $57 \pm 13$ \\
RAM15 & Transport ATPase & $203 \pm 48$ & $39 \pm 5$ \\
RAM16 & N/A & $170 \pm 40$ & $36 \pm 11$ \\
RAM17 & N/R & $211 \pm 50$ & $55 \pm 5$ \\
RAM18 & N/A & $182 \pm 43$ & $45 \pm 13$ \\
\hline
\end{tabular}

GO: N/R, no results; N/A, not applicable, intergenic insertion. Adhesion (wild type $23 \pm 8 \%$ ): $n=6$. Random motility speed (wild type $5.1 \pm 0.5 \mu \mathrm{m} / \mathrm{min}$ ) $n=3 ; 30-40$ cells (SD), ANOVA, post hoc Dunnet's: all $P<0.01$ except: ${ }^{*} P<0.05$; ${ }^{\dagger} P>0.05$ 


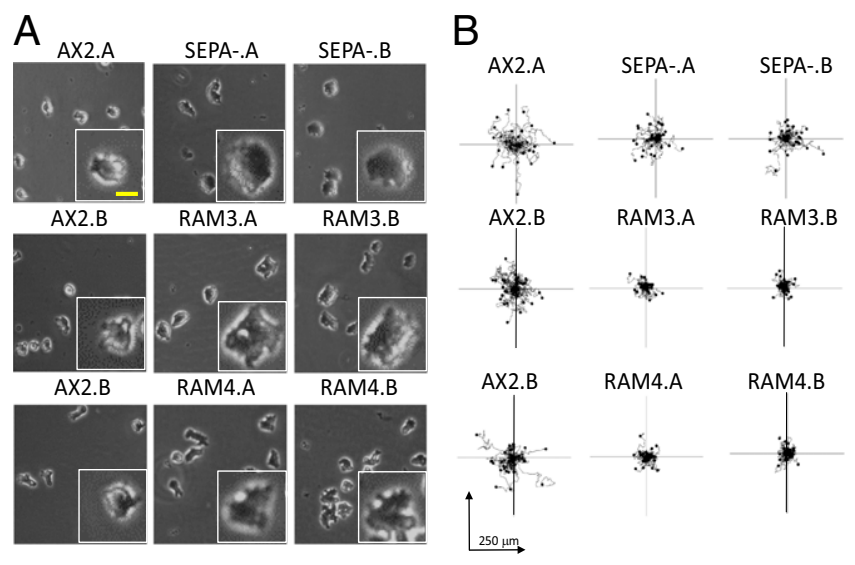

Fig. 2. Independently isolated RAM mutant and SepA deletion strains. (A) A single frame was taken from a random migration time-lapse video to show the morphology of RAM mutants or SepA deletion strains. In each case, the inset shows a closer examination of a single cell. (Yellow scale bar, $5 \mu \mathrm{m}$.) $(B)$ For one sample movie, the image sequences were analyzed, and the tracks were centered at an origin to compare random migrations. $n=30-40$ cells; the letters $A$ and $B$ indicate independent isolations of the mutant cell type.

prolonged expression of cAR1 during the developmental process, whereas pten- and RAM1, RAM4, RAM8, and RAM13 showed similar patterns of cAR1 expression as wild-type cells (SI Appendix, Fig. S4A). All of the RAM cells showed a defect in chemotaxis demonstrated by migration tracks toward a micropipette releasing cAMP, but RAM8 showed the smallest defect (SI Appendix, Fig. S4B, and Movies S8-S10). Several phenotypes arise when these RAM lines are placed on an agar surface and allowed to progress through their multicellular development (SI Appendix, Fig. S5). Both pten- and RAM3 cell lines show no developmental processes, and at $24 \mathrm{~h}$, no multicellular structures are evident. The sep $A$ strain demonstrates a similar developmental time course as wildtype cells, but many cells do not stream into mounds, and by $24 \mathrm{~h}$, fruiting bodies have only begun to form. The remaining RAM strains show a clear delay in aggregating at $8 \mathrm{~h}$ and by $24 \mathrm{~h}$ are still in mounds or formed aberrant fruiting bodies.

Validation of Specific RAM Phenotypes. For several genes, it was immediately clear that the insertions caused the phenotype because multiple independent insertions in the same gene resulted in the same phenotype (SI Appendix, Table S1). For RAM5 a single insertion was found upstream of the sepA gene, which encodes a serine/threonine kinase. Several independent REMI mutations have been reported at the same site which produced phenotypes with defects in cytokinesis and actin cytoskeleton activity (28). In addition, we created two deletion knockout mutants in which most of the sepA coding sequence was removed. The spread morphology and the decreased random migration track lengths of the sep $A$ cells are like the original RAM5 mutants (Fig. 2). The relative increases in adhesion and decrease in migration speed compared with wild type were also like the original RAM5, indicating that the phenotypic defects of RAM5 were due to the loss of SEPA (Table 2). For RAM3, we isolated eight independent insertions in an unannotated ORF. Further examination showed that the encoded protein contains eight transmembrane domains; it was predicted to be in the horizontally transferred transmembrane domain family (HTTM) by PFAM analysis, and we designated this gene htmA (SI Appendix, Table S1) (29). The similar altered morphologies and behavior of different isolates of RAM3 are shown in Fig. 2 and quantitated in Table 2. For RAM4, four insertions occurred in another unannotated ORF. Further examination of this amino acid sequence revealed Ankyrin repeats and homology to the human Ankyrin 1 gene, and we designated this gene Ankyrin Associated A
Table 2. Adhesion and motility of independent isolates

\begin{tabular}{lcc} 
Strain & \% of WT adhesion & \% of WT speed \\
\hline RAM3.1 & $181 \pm 14$ & $47 \pm 9$ \\
RAM3.2 & $188 \pm 22$ & $39 \pm 4$ \\
RAM3.3 & $220 \pm 25$ & $55 \pm 6$ \\
RAM3.4 & $205 \pm 22$ & $37 \pm 1$ \\
RAM4.1 & $306 \pm 22$ & $56 \pm 11$ \\
RAM4.2 & $331 \pm 21$ & $45 \pm 3$ \\
RAM4.3 & $264 \pm 43$ & $46 \pm 1$ \\
RAM4.4 & $287 \pm 66$ & $55 \pm 1$ \\
sepA-0.1 & $160 \pm 14$ & $69 \pm 10$ \\
sepA-0.2 & $183 \pm 16$ & $64 \pm 7$
\end{tabular}

Adhesion: $n=6$ (all SD) (WT.A: $18 \pm 3 \%$, WT.B: $21 \pm 4 \%$ ). Random motility: $n=3,30-40$ cells (WT.A:4.8 \pm 0.3 , WT.B:5.5 $\pm 0.5 \mu \mathrm{m} / \mathrm{min}$ ). ANOVA, post hoc Dunnet's: all are $P<0.01$

(araA) (SI Appendix, Table S1) (29). The similar morphologies and behavior of the different isolates of RAM4 are shown in Fig. 2 and quantitated in Table 2.

We validated RAM1, RAM8, RAM12, and RAM13 by rescuing their phenotypes by expression of the associated genes (Table 3). For RAM1 the insertion was in the ORF of the annotated gene Actobindin $\mathrm{C}$ ( $\mathrm{ABNC}$ ), a putative actin binding protein containing a $\mathrm{WH} 2$ domain. The insertion for RAM8 was localized in the ORF of the annotated Ring-Finger Zinc-Finger 113 (RNF113) gene, which has strong homology to a human gene with the same annotation (SI Appendix, Table S1). The insertion site for RAM12 was mapped in between two closely related genes which show homology to human extracellular matrix adhesion modifying tenascin proteins (SI Appendix, Table S1). We designated these two genes which are both predicted to be GPI-linked proteins, TenascinLike A and B (tnlA and tnlB) (30). The insertion for RAM13 is upstream of a gene that codes for a protein which has strong homology to the human Dual Specificity Phosphatase 19, a member of the atypical DUSP family (31) (SI Appendix, Fig. S6). We designated this gene as Dual Specificity Phosphatase A (dspA).

For each of these RAM mutations the associated genes were cloned, tagged with Green Fluorescent Protein (GFP), and then expressed from an Actin 15 promoter in the original mutant strain. As shown in Fig. $3 A$ and $B$ and Table 3, expression of each gene in its respective mutant generally reversed the phase-dark phenotype and migration defects. For RAM1 and RAM8 the rescued cells were very like wild-type cells in morphology, adhesion, and motility. For RAM12 the expression of TNLA-GFP restored motility and morphology but only partially rescued the adhesion defect. To examine this result further, the non-fluorescent-tagged TnlA and TnlB proteins were expressed in RAM12 cells. Each of these genes restored the adhesion nearly to wild-type levels suggesting that the GFP tag had a deleterious effect on function and there is shared functionality

Table 3. Reversal of adhesion and motility defects

\begin{tabular}{lcc} 
Strain: Expression & $\%$ of WT adhesion & $\%$ of WT speed \\
\hline RAM1: GFP & $216 \pm 53$ & $54 \pm 7$ \\
RAM1: AbnC-GFP & $122 \pm 24$ & $95 \pm 15$ \\
RAM8: GFP & $243 \pm 73$ & $53 \pm 10$ \\
RAM8: Rnf113-GFP & $128 \pm 33$ & $97 \pm 30$ \\
RAM12: GFP & $361 \pm 25$ & $41 \pm 4$ \\
RAM12: TnIA-GFP & $274 \pm 27^{+}$ & $85 \pm 17$ \\
RAM13: GFP & $426 \pm 50$ & $34 \pm 11$ \\
RAM13: DspA-GFP & $136 \pm 22$ & $72 \pm 9$ \\
\hline
\end{tabular}

Adhesion: $n=6$ (wild type $16 \pm 3 \%$ ) (all SD). Random motility: $n=8 ; 2$ cell lines each; $30-40$ cells (wild type $4.7 \pm 0.5 \mu \mathrm{m} / \mathrm{min}$ ). ANOVA, post hoc Dunnet's: all $P<0.01$ except ${ }^{+} P>0.01$. 
A

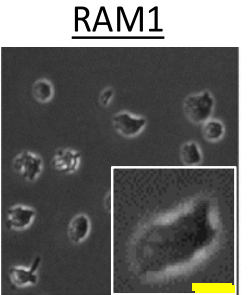

Vector-GFP

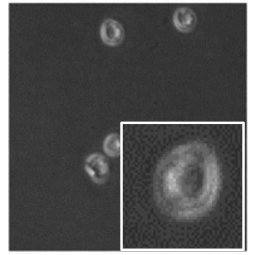

AbnC-GFP

B

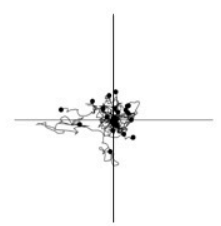

Vector-GFP

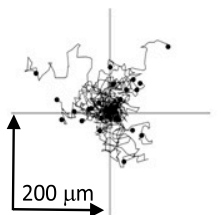

AbnC-GFP

C

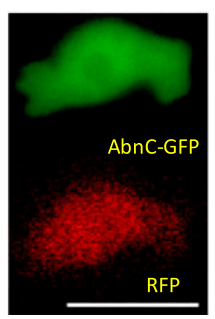

RAM8

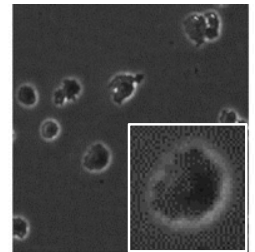

Vector-GFP

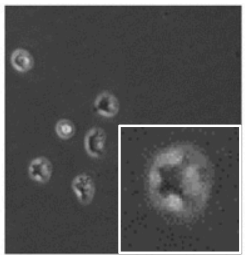

Rnf113-GFP

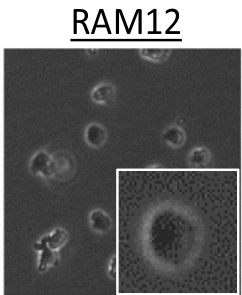

Vector-GFP

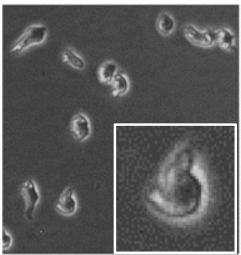

TnIA-GFP

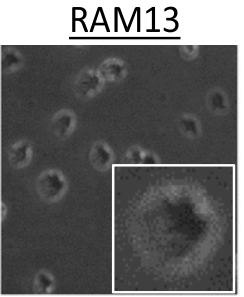

Vector-GFP

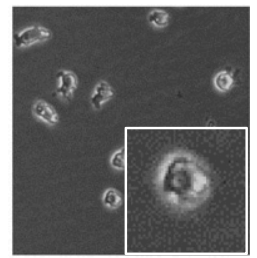

DSPA-GFP
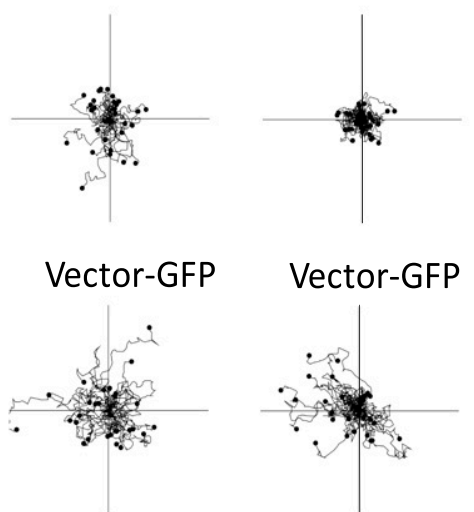

Rnf113-GFP

TnIA-GFP

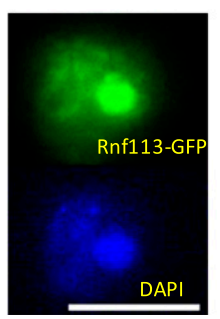

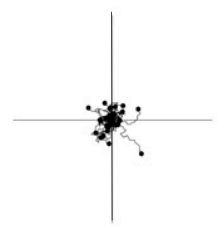

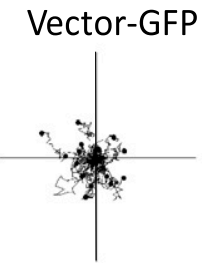

DSPA-GFP

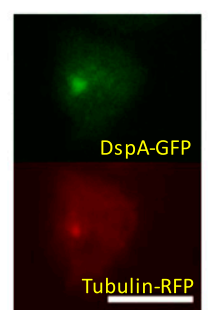

Fig. 3. Genetic rescue of RAM phenotypes and GFPtagged localizations. (A) A single frame was taken from a random migration time-lapse video to show the morphology of the indicated RAM mutants. In each case, Vector-GFP indicates the control GFP expressing vector for the cloned RAM GFP tagged gene, whereas the inset shows a closer examination of a single cell. (Yellow scale bar, $5 \mu \mathrm{m}$.) (B) For one sample video, the image sequences were analyzed, and the tracks were centered at an origin to compare random migrations. $n=30-40$ cells. (C) Each of the four cell lines rescued with the associated GFP-tagged RAM gene was imaged using epifluorescence microscopy. The RAM-GFP localizations were colocalized with an appropriate marker: AbnC with cytosolic RFP, Rnf113-GFP with nuclear localized DAPI, TnIA-GFP with membrane dye FM64, and DSPA-GFP with $\alpha$-tubulin-RFP. (Scale bars, $10 \mu \mathrm{m}$.) between these genes (SI Appendix, Fig. S7A). Expression of RAM13 rescued the adhesion and morphology defects, but the random migration phenotype was not completely mitigated. This does not appear to be a result of the overexpression of the GFP-tagged proteins, because the same construct in wild-type cells did not have a deleterious effect on migration speed (SI Appendix, Fig. S7B).

Regulators of Adhesion and Motility Are Found in Several Different Cellular Compartments. In addition to rescuing morphological and behavioral phenotypes, the functional rescues with GFP-tagged proteins allowed us to localize these proteins in living cells providing additional information on the function of these four RAM genes. AbnC-GFP appears to be localized to the cytosol. It is colocalized with a cytosolic red fluorescent protein (mRFPmars) expressed under the same promoter (Fig. 3C) (32). Most of the RNF113-GFP signal colocalized with DAPI staining indicating nuclear localization, with a fraction having a granular cytosolic localization. This localization is consistent with the Zinc-Finger domain present in the RNF113 amino acid sequence (33). TNLA-GFP is localized to the plasma membrane and cytosol with about $10 \%$ of the signal colocalized with a fluorescent plasma membrane dye (Fig. 3C). A plasma membrane localization would be expected because the TNLA and TNLB proteins are predicted to have GPI linkage (30). DSPA-GFP localized to and directly around the microtubule organizing center (MTOC) with weaker localization to the proximal regions of microtubules. A portion of the signal colocalized with $\alpha$-tubulin-mCherry. The dynamics of these four GFP-tagged proteins during migration showed no specific changes associated with active protrusions or retractions.

Cell Shape and Direct Adhesion Measurements in RAM Cells. To directly measure cell-substrate adhesion, single-cell atomic force spectroscopy measurements were taken of cells as they were pulled off the surface. Unlike the shear force adhesion assay where percentage of cells remaining is measured, this method provides direct measurements of individual cell adhesion that is not directly dependent on cell shape. We determined the work of adhesion and the number of tether-like steps upon whole-cell deadhesion events and 
A

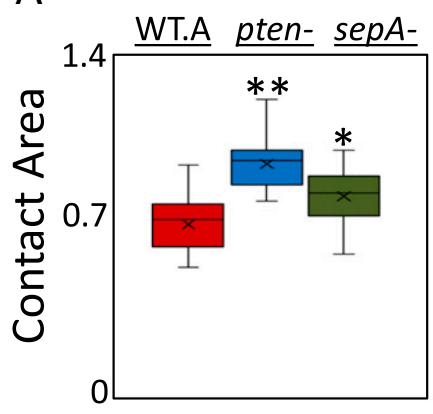

D
B

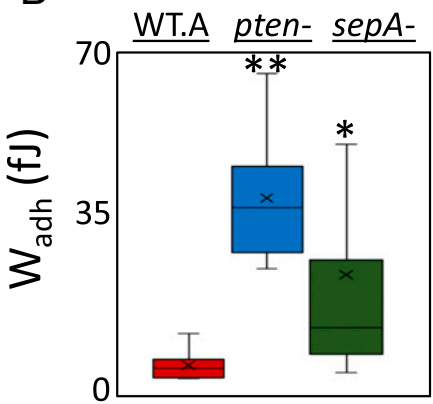

C

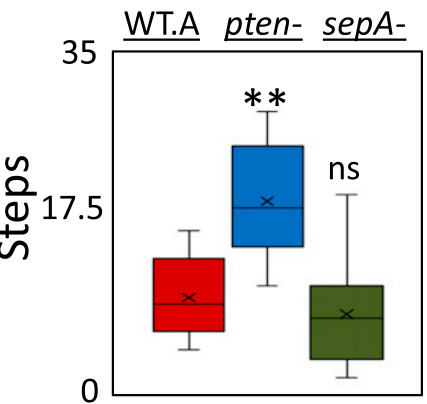

Wild Type

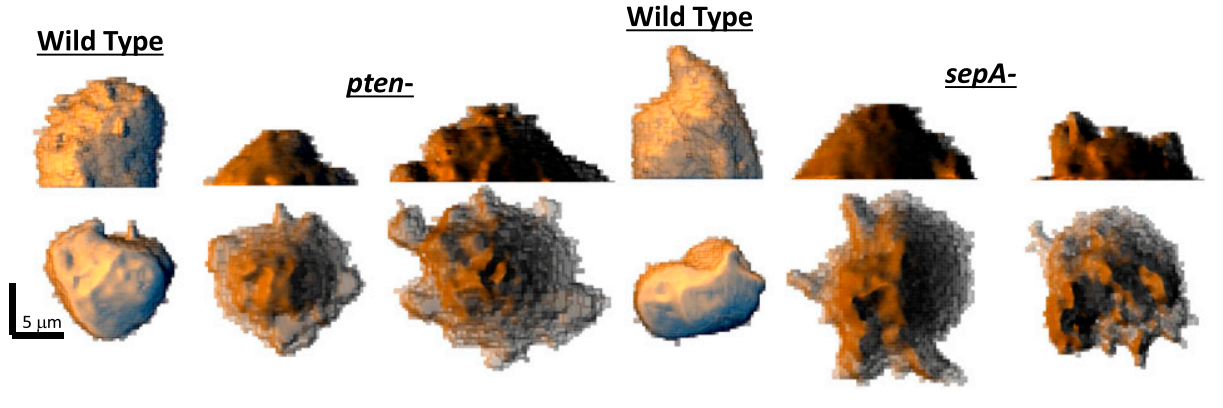

\section{E}
WT.A
pten- $\operatorname{sep} A-$
WT.B RAM 1
RAM 3
RAM 4 RAM 8
RAM 12 RAM 13

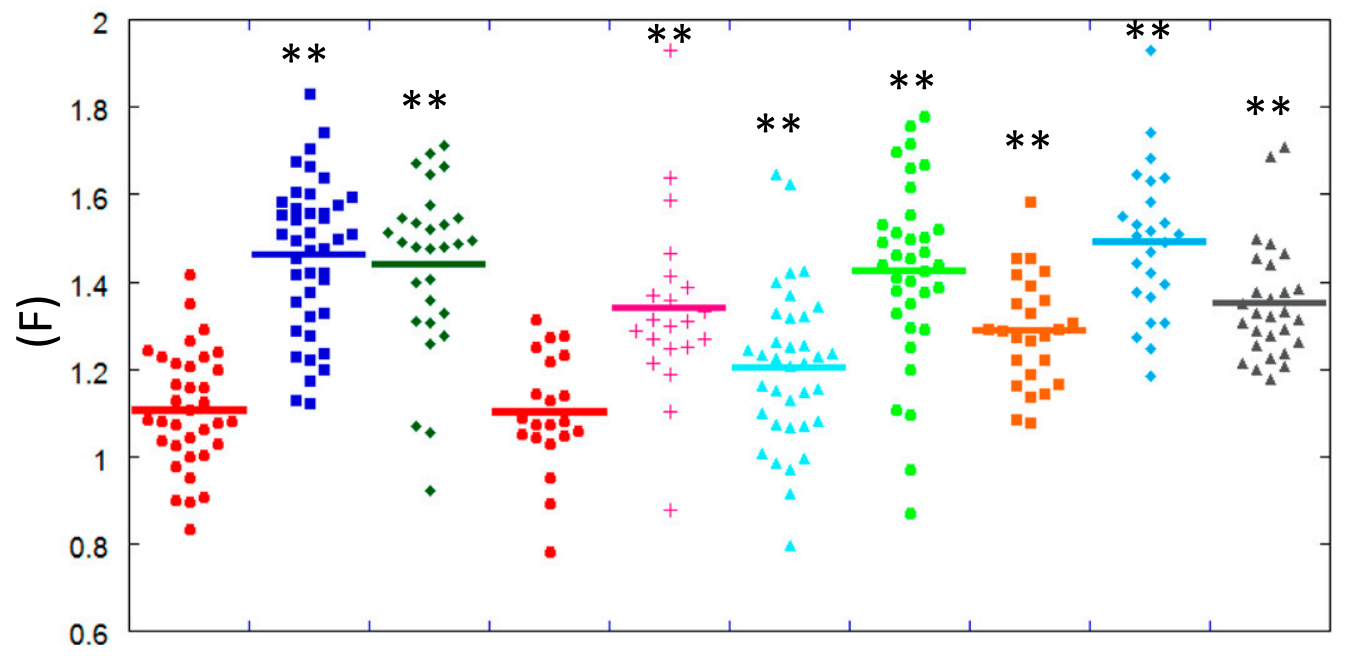

Fig. 4. Single-cell substratum adhesion and flatness of selected RAM cells. (A) Box plot of the RICM area of WT.A $(n=29)$, pten- $(n=21)$, and sepA- $(n=21)$ cells. $(B)$ Box plot of the work of the adhesion $\left(W_{\mathrm{adh}}\right)$ of WT.A $(n=62)$, pten- $(n=20)$, and sepA- $(n=81)$ cells. (C) Box plot of the steps recorded during deadhesion of WT.A $(n=23)$, pten- $(n=20)$, and sepA- $(n=94)$ cells. In all three box plots $(A-C)$, quartile calculation is exclusive median, $x$ represents the mean, Wilcoxon rank-sum test. $(D)$ Representative cells from the indicated strains shown in both a lateral and ventral views. $(E)$ Using the FM-64 membrane dye, the indicated RAM mutants have been analyzed for shape and size. Spinning disk confocal Z-stacks were taken and analyzed using Image J. $n=20-$ 30 cells; line represents the mean. Flatness parameters defined as the basal area's square root divided by the volume's cubed root. ANOVA post hoc Dunnet's test, ${ }^{*} P<0.05, * * P<0.01$; not significant (ns), $P>0.05$.

related the cell adhesion to contact area as displayed in reflection interference contrast imaging. In this assay the positive control ptencells have a clear increase in adhesion measured as work required to completely remove the cell from the substrate and show a significantly increased contact area (Fig. $4 A$ and $B$ ) (SI Appendix, Fig. $\mathrm{S} 8 A$ ). Furthermore, they displayed an elevated number of steps, reflecting an increase in specific substrate anchoring sites and the corresponding lipid cylinder protrusions formed upon deadhesion (Fig. 4C) (34). The newly generated knockout $\operatorname{sep} A$ - also shows an increase in work of adhesion and contact area. The sep $A$ - cells do not show a significant increase in the number of adhesion tethering sites.
These results suggest that most the newly isolated RAM strains might have directly increased cell-substrate adhesion, increased contact area, and/or increased substrate binding sites.

We created 3D reconstructions of cells stained with a fluorescent membrane dye to assess whether the phase-dark appearance and their increased contact area was leading to a flattened cell morphology. As shown by the 3D reconstructions in Fig. $4 D$, our imaging method could clearly demonstrate that pten- and $\operatorname{sep} A$ mutants were flatter than wild-type cells. To quantitate this, we used a flatness parameter $(F)$ using the ratio of the square root of the basal surface area $(a)$ to the cube root of the volume $(v)$ 


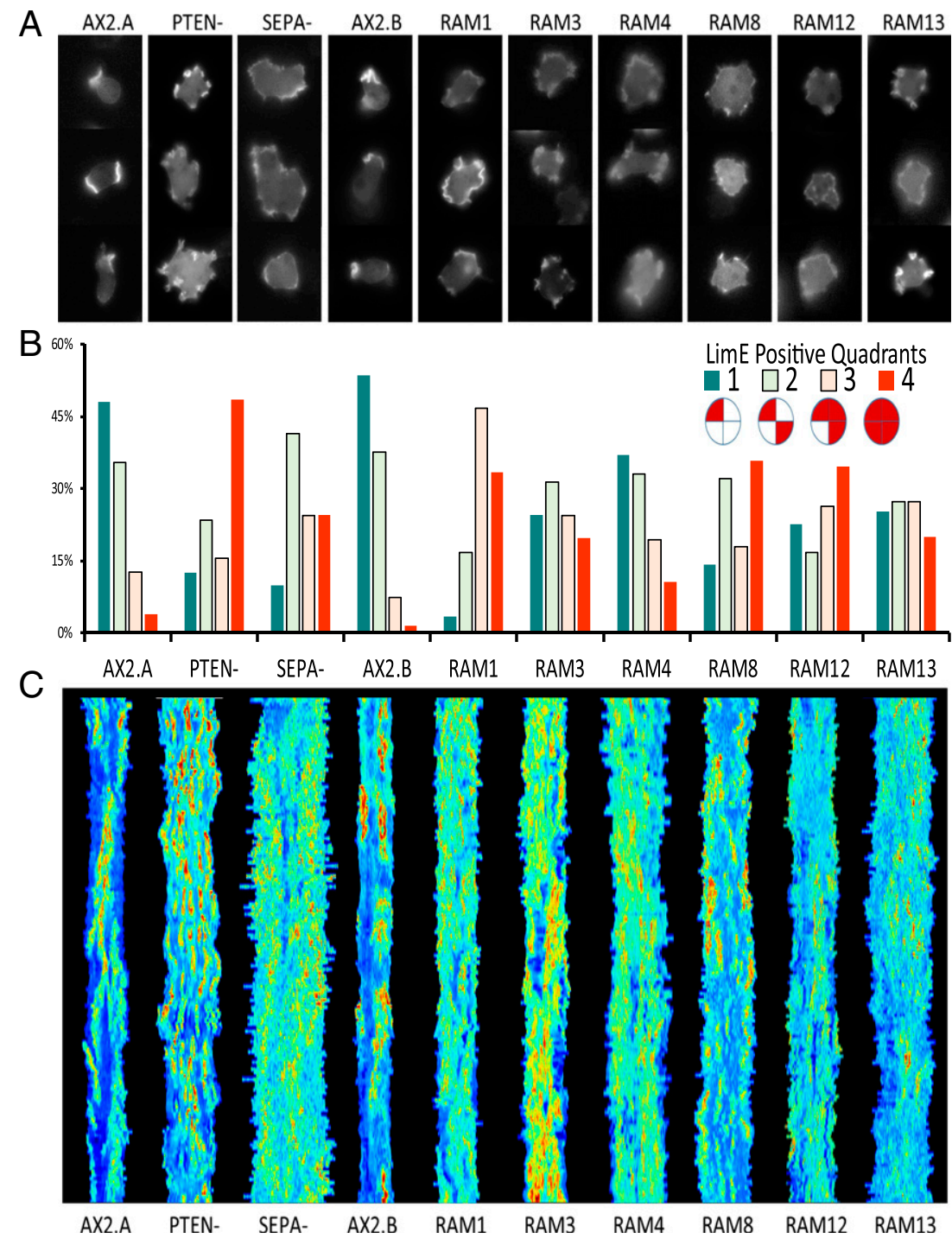

Fig. 5. Actin dynamics in confirmed RAM mutant strains expressing LimE $_{\Delta \text { coil }}$ RFP. (A) Representative examples of vegetative cells depicting the actin-based protrusions compared with wild type and PTEN- cells. Many of the RAM mutants display multidirected LimE $_{\Delta \text { coil }}-R F P$ positive patches on their membrane. Brightness and contrast of the images were adjusted show similar expression of the bioprobe. (B) These images were quantified by determining the quadrants positive for actin-based protrusions. $n=3$ experiments, 20-30 cells each. (C) Time-lapse videos were taken of these cells and then analyzed using a MATLAB-based algorithm to create kymographs of the LimE $_{\Delta c o i l}-\mathrm{RFP}$ protrusion dynamics. Time-lapse movies depicted in kymographs are from 6-s frame intervals for $20 \mathrm{~min}$. Color in the kymograph is representative of the scaled membrane signal: blue $\rightarrow$ red $=0 \rightarrow 100 \%$ intensity.

$\left(F=a^{1 / 2} / v^{1 / 3}\right)(35) . F$ is independent of the object's volume and can be compared across different cell types. As shown in Fig. $5 E$ the average values for $F$ for wild-type and pten- cells are $1.11 \pm$ 0.13 and $1.46 \pm 0.17$, respectively, again demonstrating that ptencells are flatter than their wild-type parent control. The $F$ values for the RAM mutants were all significantly greater than those of the parental wild-type cells. There were only slight differences in the volumes of the RAM cells, except RAM8 which is generally smaller, but the most RAM basal surface areas are greater which leads to their increase in $F$ (SI Appendix, Fig. S8 $B$ and $C$ ). Taken together these suggest that adhesion and fluctuations in the cellsubstrate distance are a sensitive measure for overall morphology and motility (36-38).

Cytoskeletal Network Activities Reveal How the RAM Genes Might Regulate Cell-Substrate Adhesion and Motility. We next assessed whether the spreading and flattening, as well as the increase in tether-like rupture events of the RAM cells, were driven by increased F-actin protrusions along the periphery of the cells. Cells were transformed with $\mathrm{LimE}_{\Delta \text { coil- }} \mathrm{RFP}$, a biosensor for newly formed F-actin (39). As shown in Fig. 5A, unlike wild-type cells which generally had a single front, most RAMs had actin-filled protrusions at multiple positions around the cell perimeter. Images of cells were quantified by dividing each cell into quadrants, scoring the number of quadrants occupied with $\mathrm{LimE}_{\Delta \text { coil- }} \mathrm{RFP}$ positive protrusions, and determining the fraction of the population with one to four occupied quadrants. As anticipated, the distributions of wild-type and pten- cells were strongly skewed in opposite directions with $45 \%$ or $4 \%$ of the wild-type cells having one or four quadrants occupied by LimE signal, respectively, and pten- cells having $12 \%$ or $45 \%$. Although there was a spectrum of the distributions for the RAM mutants, the quantitation clearly showed that compared with wild-type cells, RAMs mutants have more quadrants occupied by signal simultaneously (Fig. $5 B$ ). To illustrate the dynamics of the protrusions in wild-type and mutant cells, we generated kymographs of the intensity of $\operatorname{LimE}_{\Delta \text { coil-RFP }}$ on the cell perimeter (Fig. $5 C$ ). In wild-type cells, there are typically one to two patches of F-actin at a given time, which last for $\approx 2-3$ min. In ptenand RAM8 cells, there are smaller shorter-lived protrusions that occupy multiple regions of the cell periphery. In the remaining RAM mutants, it appears that the LimE signal is present in several directions simultaneously with a more stable but weaker signal, sometimes lasting for $20 \mathrm{~min}$. Both cytoskeletal activity patterns would lead to increased spreading. This nonpolarized F-actin production in the RAM cells also explains the decreased random migration velocities. Finally, the increased F-actin along the periphery of the cells and increased spreading likely underlies the increased 
cell-substrate adhesion and could facilitate tether formation in RAM mutants.

Phenotypes of the RAMs Mutants Are Strongly Correlated and Dependent on Signal Transduction Network Activities. We reasoned that the observed F-actin enrichment might be driven by an increase in upstream signal transduction activity. The Dictyostelium PKB homologs PKBA and PKBR1 play a critical role in actin assembly and migration. We used antibodies against the phosphorylated consensus motifs of PKB to determine the baseline levels of PKB activity in RAM cells and their parental strains. We observed significant changes in baseline phosphorylation for several PKB substrates (SI Appendix, Fig. S9C). In Fig. $6 C$ and $D$ the average intensity of three bands, pp $100 \mathrm{kDa}, \mathrm{pp} 85 \mathrm{kDa}$, and pp $19 \mathrm{kDa}$, is shown. Substrate phosphorylation was 2.5 -fold higher in pten- compared with the corresponding parental strain, likely due to increased PIP3 levels and PIP3-dependent activation of PKBA (40). RAM1, 4, 8, 12, and 13 also showed elevated PKB substrate phosphorylation across the multiple substrates (Fig. $6 C$ and $D$ ). Notably, these observations correlate with the increased F-actin accumulation in these RAM cells shown in Fig. 5.
Because the small G protein Rap1 has been demonstrated to provide a supportive role in Dictyostelium protrusion generation and substrate adhesion, we examined how the expression of the dominant negative Rap1S17N-myc would affect adhesion in the confirmed RAM strains (41-45). In wild-type cells, Rap1S17N has been shown to increase myosin IIB at the cell cortex which leads to cell retraction and decreased adhesion (44). As shown in Fig. $6 A$, expression of Rap1S17N in our wild-type strain did led to a more retracted shape with smaller protrusions. The two knockout lines sep $A$ - and pten- and most of the RAM strains also shift from their flattened phase dark appearance to a brighter, retracted appearance when expressing Rap1S17N, although most did not retract to the same degree as wild-type cells. However, RAM12 cells had almost no response to Rap1S17N expression maintaining most of its phase-dark appearance. These morphological changes correlate to a decrease in adhesion (Fig. $6 B)$. All of the cell lines examined expressed Rap1S17N-myc (SI Appendix, Fig. S9B).

\section{Discussion}

The first 18 unique characterized insertional mutation strains isolated from these procedures fall into a spectrum of pheno-
A
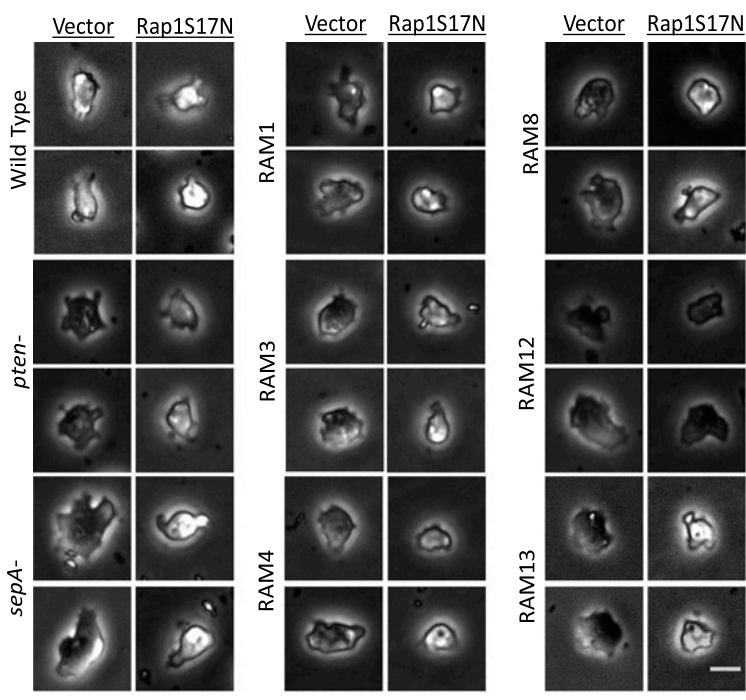

B

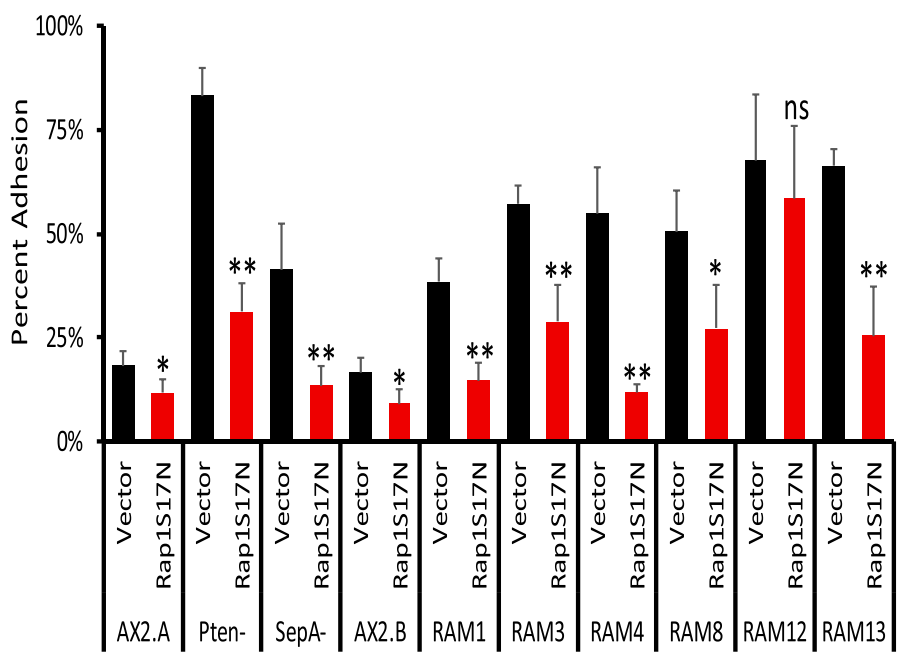

C
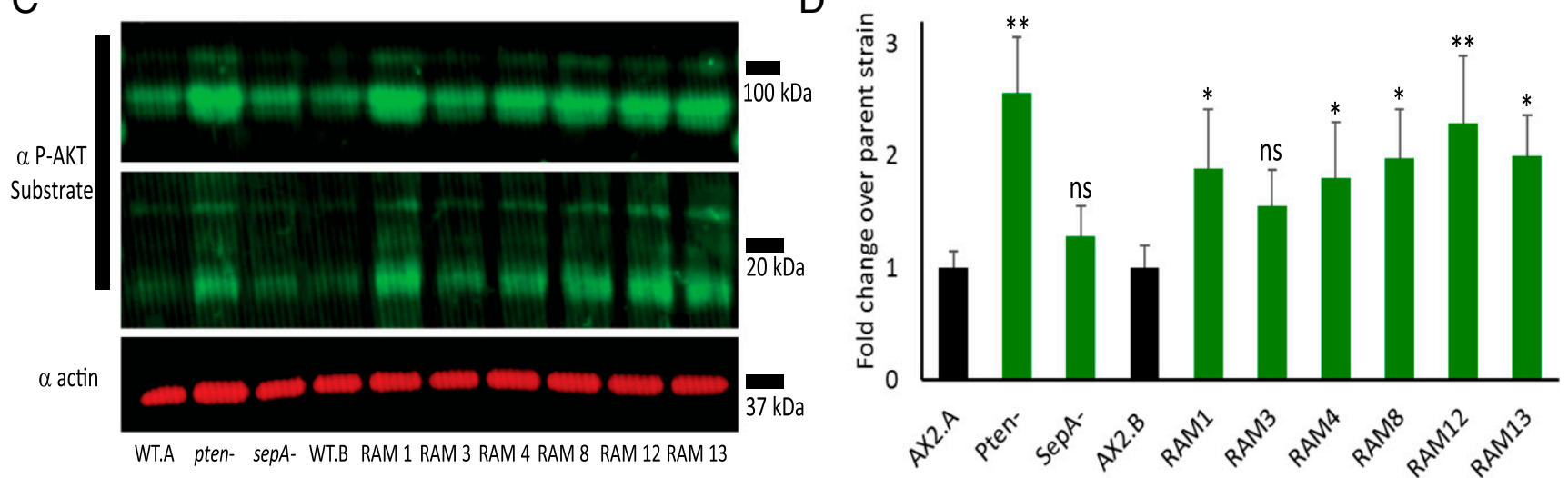

Fig. 6. Suppression of RAM mutant phenotypes with dominant negative Rap1 and analyses of phospho-AKT activation. ( $A$ ) Representative phase contrast images of the select RAM cell types expressing control vector or overexpressing dominant negative Rap1S17N. (Scale bar, $10 \mu \mathrm{m}$.) (B) The shear forced adhesion assay was performed on these cells at $150 \mathrm{rpm}$ for $1 \mathrm{hr}$ showing reduced adhesion in RAM cells. $n=6$, Student's $t$ test. (C) Representative immunoblot for P-AKT substrates (green) and actin control (red), $1 \times 10^{6}$ cells loaded per lane. $(D)$ The averages of the p100kDA, p85kDA, and p19kDA bands were normalized to the respective wildtype parent strain. $n=4$, ANOVA post hoc Dunnet's test $* P<0.05, * * P<0.01$; not significant (ns), $P>0.05$. 


\section{Cell Migration Cell-Substrate Adhesion}

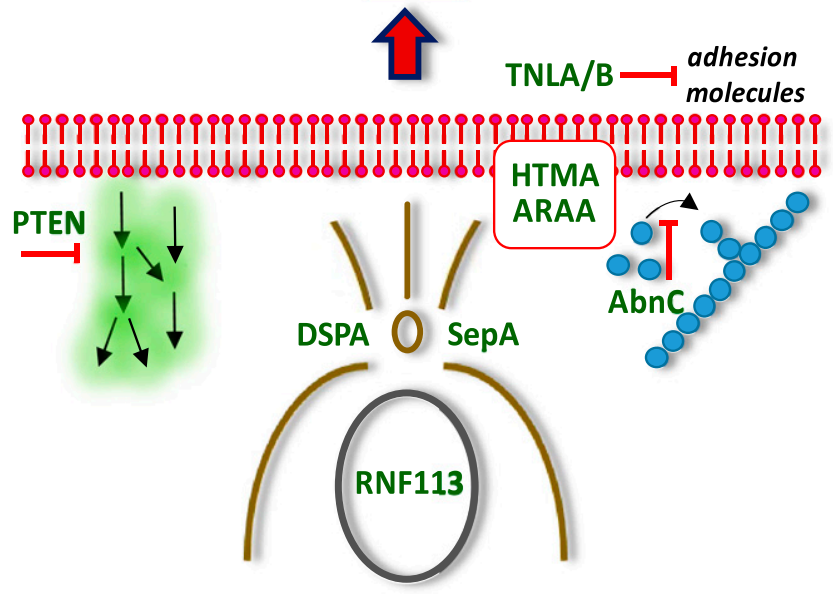

Fig. 7. Cell-substrate adhesion and migration have multiple regulatory points. PTEN and the seven confirmed RAM genes are illustrated in their theoretical position in controlling adhesion and migration. PTEN is known to function in controlling the signaling cascades depicted by the black arrows. The red lines in the middle represent the plasma membrane and the position in which TNLA/B, HTMA, and ARAA are predicted to function. Both SepA and DSPA are localized to the centrosome. RNF113 has been shown to have at least localization to the nucleus (and the cytosol). AbnC is localized in the cytosol but is predicted to regulate the actin cytoskeleton depicted by the blue boxes.

types between wild-type and pten- cells of increased adhesion and decreased migration. All of the seven mutants that we chose to initially validate, because they were in the top half of the adhesion profile, showed that loss of the function of the genes associated with the REMI insertions underlie the reported phenotypes. These mutants are flatter, have increased polymerized actin along their peripheries, and are defective in chemotaxis. Many of them have increased signal transduction activity, and almost all adhesion and morphological phenotypes can be suppressed by the introduction of dominant negative Rap1. The spectrum of genetic ontologies and subcellular localizations discovered using this screen suggests that there are multiple points of regulation of adhesion and migration. Our method will likely uncover many other genes regulating cellular adhesion and migration, among the strains we have already isolated but not yet characterized, and in further screens. Because adhesion and migration appear to be coupled, it might be easier to identify and select for adhesion as opposed to looking for motility differences in a population.

The increased adhesion of RAM cells could be due to a direct effect of specific adhesion molecules, increased surface area coupled with nonspecific adhesion, or both. We have found evidence for both mechanisms due to an increase in tether and contact area. Dictyostelium cells express several substrate adhesions regulating molecules including Phg1, SadA, and an ortholog of integrin $\beta$-subunits designated like integrin $\beta$ A (SibA) (46-49). Dictyostelium cells are not known to form stable focal adhesions although they express homologs of talin, vinculin, and paxillin which localize to the basal surface in puncta with F-actin $(1,50-52)$. Because Dictyostelium cells need to migrate over a variety of surfaces in their natural environments, their substrate adhesion is thought to be largely nonspecific (53-55). For the seven validated mutants reported there was a strong correlation between flatness and adhesion. A nonspecific adhesion mechanism would therefore be sufficient for this effect (56). However, preliminary evidence from other RAM mu- tants (RAM6 and RAM14) indicates that some associated genes may regulate adhesion without changes in cell shape.

Regulators of Adhesion and Motility Reveal Multiple Points of Regulation. One of the genes is an Actobindin family member (ABNC); this family has been reported to be G-actin sequestering proteins that negatively regulate actin polymerization and stabilize actin filaments (57-60). However, at certain concentrations it may also assist polymerization (61). Outside of mammals which use Thymosin $\beta 4$, it appears that most eukaryotic cells use other members of the Actobindin family to regulate actin filament stability and polymerization. The amino acid sequences of the three Abn proteins (A, B, and C) in Dictyostelium differ, but because they all contain the conserved Actobindin family WH2 domain they are predicted to sequester G-actin (SI Appendix, Fig. S10). When at least one of these genes is disrupted, it leads to increased F-actin production and cell spreading which seems consistent with the expected role. This effect would constitute a direct effect on the cytoskeleton, and the fact that AKT substrate phosphorylation is elevated in this mutant suggests a feedback mechanism between the cytoskeleton and the signal transduction networks.

The $\operatorname{sep} A$ gene is a kinase that is has been reported to be part of the STE20 family, closely related to the Cdc7 in Saccharomyces pombe (62). This gene has been shown to be critical in the actin regulation during septation (63). This protein has similarity to other MAP4K family members in the human genome such as MSTs and PAKs. The closest relative of SepA in the human genome is MAP3K19 which has highest expression in the lungs and trachea (64) (SI Appendix, Fig. S11). The Dictyostelium SepA gene has previously been reported to have an effect on cytokinesis in Dictyostelium with some evidence to increased actin patches along the periphery (28).

The Dictyostelium Rnf113 protein localizes to the nucleus and has a Zinc-Finger domain and RING-Finger domain which are each greater than $81 \%$ and $53 \%$ identical, respectively, to human RNF113b. The RING-Finger domain in RNF113a has been shown to be mutated in a subset of the human disease trichothiodystrophy nonphotosensitive, up-regulated in Myelodysplastic syndrome, and phosphorylated in prostate cancer (6567). Currently, the function of this protein remains mostly elusive with some evidence suggesting regulation of DNA repair and splicing mechanisms $(68,69)$. Work in Caenorhabditis elegans, Drosophila melanogaster (midlife crisis), and Danio rerio has also provided evidence for developmental defects (70-73). The RING-Finger domain of this protein falls into the same family as the one contained in BRCA1, and there is evidence that BRCA1 regulates motility at the leading edge beyond its role of DNA repair in the nucleus (74).

TnlA and TnlB are predicted to be GPI-linked peripheral membrane proteins, and consistently, we localized them to the plasma membrane. These two proteins share homology to the highly glycosylated tenascins in humans. Remarkably, their disruption creates a strong phenotype even though each is only a single member of a family of over 100 related genes in Dictyostelium (Linnaeus BLAST: 8e-7) (75). Tenascins are reported to negatively regulate cell interactions with the extracellular matrix, and overexpression has been shown to increase metastasis from primary tumors (76). Based on these findings we hypothesize that TnlA and TnlB may act at the interface of the cell and the substratum to directly affect cell adhesion and migration. Consistently, this increased adhesion was not suppressed by inhibition of signal transduction activity by expression of dominant negative Rap1.

Dictyostelium only possess a single atypical DUSP compared with the 22 genes present in humans. Moreover, the DSPA amino acid sequence is most closely related to the more ancestral human atypical DUSPs (SI Appendix, Fig. S6). This subfamily of 
the tyrosine phosphatase family has been reported to negatively regulate MAPK and AKT pathway and is closely related to inositol phosphatases such as PTEN (31). Moreover, the aDUSPs have been shown to play a role in regulating adhesion through the JNK pathway (77). Like DSPA, the closely related human DUSP23 is also localized to the centrosome further suggesting conserved functions (78). Moreover, the DSPA related human DUSP22 has been shown to play a role in epithelial migration by scratch wound assay (12). Because this is the only member of the aDUSP family in Dictyostelium, its study will provide important insights into the poorly understood functions that these proteins play in cell migration.

RAMs Negative Regulators of Cell Activity and Tumor Suppressors. The large spectrum of RAM genes indicates that cell-substrate adhesion and migration can be regulated at many different levels by many different cellular pathways and networks. As depicted in Fig. 7 we speculate that PTEN interacts with the signal transduction network, TNLA/B regulate cell-substrate interactions at the cell surface, SepA and DSPA regulate phosphorylation at the microtubule network, RNF113 functions inside the nucleus, and the $\mathrm{AbnC}$ acts in the cytosol to regulate actin polymerization. The localization of the remaining two genes remains to be assigned, but HTMA is most likely an integral membrane protein, whereas ARAA is possibly a cytosolic or peripheral membrane protein.

It is remarkable that most of these proteins feed into the signal transduction and cytoskeletal networks known to be involved in chemotaxis. Specifically, we found that there is an increased level of activity of AKT homolog PKBA in most of these mutant strains. It will be interesting to learn how, for example, proteins localized to the cytosol, plasma membrane, MTOC, or the nucleus can all negatively regulate PI3K pathways. Two of the RAM proteins localizing to the MTOC (SepA and DSPA) provide further support for the importance of microtubules in regulating cell polarity, migration, and adhesion (28, 79-81). Moreover, almost all of the phenotypes can be suppressed by overexpression of dominant negative Rap1 which is thought to be a regulator in multiple pathways involved in chemotaxis. In Dictyostelium, Rap1 has been reported to either activate Ras or negatively regulate myosin leading to increased $\mathrm{PKB}$ signaling and decreased contraction $(41,42,44)$. These mutants show increased patches of actin polymerization suggesting that directly or indirectly, the functions of the RAM proteins feed into negatively regulating cytoskeletal activity. The net result of these effects is to create a spread cell with increased cytoskeletal and signal transduction activity, leading to increased adhesion and decreased random and directed migration.

1. Veltman DM, King JS, Machesky LM, Insall RH (2012) SCAR knockouts in Dictyostelium: WASP assumes SCAR's position and upstream regulators in pseudopods. J Cell Biol 198:501-508

2. Rocheleau AD, Sumagin R, Sarelius IH, King MR (2015) Simulation and analysis of tethering behavior of neutrophils with pseudopods. PLoS One 10:e0128378.

3. Insall R (2013) The interaction between pseudopods and extracellular signalling during chemotaxis and directed migration. Curr Opin Cell Biol 25:526-531.

4. Pollard TD, Borisy GG (2003) Cellular motility driven by assembly and disassembly of actin filaments. Cell 112:453-465.

5. Eddy RJ, Pierini LM, Matsumura F, Maxfield FR (2000) Ca2+-dependent myosin II activation is required for uropod retraction during neutrophil migration. J Cell Sci 113:1287-1298.

6. Noegel AA, Schleicher M (2000) The actin cytoskeleton of Dictyostelium: A story told by mutants. J Cell Sci 113:759-766.

7. Moores SL, Sabry JH, Spudich JA (1996) Myosin dynamics in live Dictyostelium cells. Proc Natl Acad Sci USA 93:443-446.

8. Huang CH, Tang M, Shi C, Iglesias PA, Devreotes PN (2013) An excitable signal in tegrator couples to an idling cytoskeletal oscillator to drive cell migration. Nat Cell Biol 15:1307-1316.

9. Artemenko Y, Lampert TJ, Devreotes PN (2014) Moving towards a paradigm: Common mechanisms of chemotactic signaling in Dictyostelium and mammalian leukocytes. Cell Mol Life Sci 71:3711-3747.

10. Tang M, et al. (2014) Evolutionarily conserved coupling of adaptive and excitable networks mediates eukaryotic chemotaxis. Nat Commun 5:5175.
Although most of the mutants show defects in their chemotactic velocity, this is most likely due to decreased migratory abilities and not altered directional sensing. Although the directional signal probably lies upstream with the receptor and coupled $G$ proteins, the downstream signal transduction networks are essential in connecting the directional signal to movement. If the basal activity of the downstream pathways is increased by the loss of negative regulation, as in the RAM mutants, then the directional signal relative to the noise is lower, and the ability to specifically direct cytoskeletal based protrusions is diminished. Therefore, genetic screens that isolate genes important for regulating cell-substrate adhesion and random migration will also discover genes important for the overall process of chemotaxis.

Previous research has shown many regulators of chemotaxis pathways in Dictyostelium have direct homologs to human oncogenes and tumor suppressors $(9,14)$. Because we used the tumor suppressor PTEN as a positive control, this screen has the potential to identify genes whose human homologs play a role in oncogenesis. In fact, the isolation of Dictyostelium Abns, SepA, TNLA/B, and DSPA has already suggested that this may be the case. Thymosin $\beta 4$, a human member of the Actobindin family, has been reported to play the role of both an oncogene and tumor suppressor under different contexts $(82,83)$. SepA shares homology with human MSTs and PAKs, known tumor supressors that are currently being investigated as therapeutic targets $(84,85)$. Human Tenascin- $\mathrm{C}$ is reported to play a role in the progression and metastasis of breast cancer and as a potential target $(86,87)$. The human DUSP proteins have been reported to play numerous roles in oncogenesis and cancer progression, are useful diagnostic markers, and are promising drug targets (88-90).

\section{Materials and Methods}

For all experiments, Dictyostelium discoideum AX2 strains were cultured in HL5 medium on tissue culture treated plastic 100-mm (Greiner Cellstar) or $150-\mathrm{mm}$ plates (Falcon) for $48 \mathrm{~h}$ to $80-90 \%$ confluency at $22{ }^{\circ} \mathrm{C}$ (91). Fluorescent imaging and chemotaxis assays were performed in developmental buffer (DB) (phosphate buffer supplemented with $2 \mathrm{mM} \mathrm{MgSO}_{4}$ and $0.2 \mathrm{mM}$ $\mathrm{CaCl}_{2}$ ). Complete materials and methods can be found in SI Appendix, Materials and Methods.

ACKNOWLEDGMENTS. We thank Pablo Iglesias for his help in supplying and modifying his MATLAB algorithms for the creation of the kymographs. We thank Christopher Janetopoulos for his initial help characterizing the RAM12 mutant and making the TnIA expression constructs. We thank Yulia Artemenko and Nayai Hill for their initial isolation and characterization of the sepA-strains. We thank the Johns Hopkins University School of Medicine Microscope Facility for their expert help in many of the microscope experiments presented in the research. This study was supported by National Institute of Health R35 GM118177 (to P.N.D.), American Cancer Society 124959-PF-13-178-01-CSM (to T.J.L.), and Deutsche Forschungsgemeinschaft Grant SFB 937 subproject A8.

11. Cram EJ, Shang H, Schwarzbauer JE (2006) A systematic RNA interference screen reveals a cell migration gene network in C. elegans. J Cell Sci 119:4811-4818.

12. Simpson KJ, et al. (2008) Identification of genes that regulate epithelial cell migration using an siRNA screening approach. Nat Cell Biol 10:1027-1038.

13. Lesch C, Jo J, Wu Y, Fish GS, Galko MJ (2010) A targeted UAS-RNAi screen in Drosophila larvae identifies wound closure genes regulating distinct cellular processes. Genetics 186:943-957.

14. Swaney KF, Huang CH, Devreotes PN (2010) Eukaryotic chemotaxis: A network of signaling pathways controls motility, directional sensing, and polarity. Annu Rev Biophys 39:265-289.

15. Balla T (2013) Phosphoinositides: Tiny lipids with giant impact on cell regulation. Physiol Rev 93:1019-1137.

16. Parent CA, Devreotes PN (1999) A cell's sense of direction. Science 284:765-770.

17. Servant G, et al. (2000) Polarization of chemoattractant receptor signaling during neutrophil chemotaxis. Science 287:1037-1040.

18. Cozier GE, Carlton J, Bouyoucef D, Cullen PJ (2004) Membrane targeting by pleckstrin homology domains. Curr Top Microbiol Immunol 282:49-88.

19. lijima M, Devreotes $P$ (2002) Tumor suppressor PTEN mediates sensing of chemoattractant gradients. Cell 109:599-610.

20. Artemenko Y, Swaney KF, Devreotes PN (2011) Assessment of development and chemotaxis in Dictyostelium discoideum mutants. Methods Mol Biol 769:287-309.

21. Huber RJ (2014) The cyclin-dependent kinase family in the social amoebozoan Dictyostelium discoideum. Cell Mol Life Sci 71:629-639. 
22. Cao L, et al. (2014) Phylogenetic analysis of CDK and cyclin proteins in premetazoan lineages. BMC Evol Biol 14:10.

23. Lemmon MA, Freed DM, Schlessinger J, Kiyatkin A (2016) The dark side of cell signaling: Positive roles for negative regulators. Cell 164:1172-1184.

24. lijima M, Huang YE, Luo HR, Vazquez F, Devreotes PN (2004) Novel mechanism of PTEN regulation by its phosphatidylinositol 4,5-bisphosphate binding motif is critica for chemotaxis. J Biol Chem 279:16606-16613.

25. Tang $\mathrm{M}$, et al. (2011) Disruption of PKB signaling restores polarity to cells lacking tumor suppressor PTEN. Mol Biol Cell 22:437-447.

26. Huang C, Rajfur Z, Borchers C, Schaller MD, Jacobson K (2003) JNK phosphorylates paxillin and regulates cell migration. Nature 424:219-223.

27. Asano Y, et al. (2004) Keratocyte-like locomotion in amiB-null Dictyostelium cells. Cell Motil Cytoskeleton 59:17-27.

28. Müller-Taubenberger A, Ishikawa-Ankerhold HC, Kastner PM, Burghardt E, Gerisch G (2009) The STE group kinase SepA controls cleavage furrow formation in Dictyostelium. Cell Motil Cytoskeleton 66:929-939.

29. Punta M, et al. (2012) The Pfam protein families database. Nucleic Acids Res 40: D290-D301

30. Chou KC, Shen HB (2007) MemType-2L: A web server for predicting membrane proteins and their types by incorporating evolution information through Pse-PSSM. Biochem Biophys Res Commun 360:339-345.

31. Patterson KI, Brummer T, O'Brien PM, Daly RJ (2009) Dual-specificity phosphatases: critical regulators with diverse cellular targets. Biochem J 418:475-489.

32. Fischer M, Haase I, Simmeth E, Gerisch G, Müller-Taubenberger A (2004) A brilliant monomeric red fluorescent protein to visualize cytoskeleton dynamics in Dictyostelium. FEBS Lett 577:227-232.

33. Yamasaki $\mathrm{H}$, et al. (2005) Zinc finger domain of Snail functions as a nuclear localization signal for importin beta-mediated nuclear import pathway. Genes Cells 10:455-464.

34. Lagüe P, Zuckermann MJ, Roux B (2001) Lipid-mediated interactions between intrinsic membrane proteins: Dependence on protein size and lipid composition. Biophys J 81:276-284.

35. Vogel S (2013) Comparative Biomechanics: Life's Physical World (Princeton University Press, Princeton, NJ), pp 47-48.

36. Leonhardt $\mathrm{H}$, et al. (2016) Cell-substrate impedance fluctuations of single amoeboid cells encode cell-shape and adhesion dynamics. Phys Rev E Stat Nonlin Soft Matter Phys 93:012414.

37. Schäfer E, et al. (2013) Chemotaxis of Dictyostelium discoideum: Collective oscillation of cellular contacts. PLOS One 8:e54172.

38. Tarantola M, et al. (2010) Dynamics of human cancer cell lines monitored by electrical and acoustic fluctuation analysis. Integr Biol 2:139-150.

39. Schneider N, et al. (2003) A Lim protein involved in the progression of cytokinesis and regulation of the mitotic spindle. Cell Motil Cytoskeleton 56:130-139.

40. Kamimura Y, et al. (2008) PIP3-independent activation of TorC2 and PKB at the cell's leading edge mediates chemotaxis. Curr Biol 18:1034-1043.

41. Plak K, Pots H, Van Haastert PJ, Kortholt A (2016) Direct Interaction between TalinB and Rap1 is necessary for adhesion of Dictyostelium cells. BMC Cell Biol 17:1.

42. Kortholt A, et al. (2006) Characterization of the GbpD-activated Rap1 pathway regulating adhesion and cell polarity in Dictyostelium discoideum. J Biol Chem 281:23367-23376.

43. Rebstein PJ, Weeks G, Spiegelman GB (1993) Altered morphology of vegetative amoebae induced by increased expression of the Dictyostelium discoideum rasrelated gene rap1. Dev Genet 14:347-355.

44. Jeon TJ, Lee DJ, Merlot S, Weeks G, Firtel RA (2007) Rap1 controls cell adhesion and cell motility through the regulation of myosin II. J Cell Biol 176:1021-1033.

45. Seastone DJ, et al. (1999) The small Mr Ras-like GTPase Rap1 and the phospholipase C pathway act to regulate phagocytosis in Dictyostelium discoideum. Mol Biol Cell 10:393-406.

46. Casademunt E, Varney TR, Dolman J, Petty C, Blumberg DD (2002) A gene encoding novel anti-adhesive protein is expressed in growing cells and restricted to anteriorlike cells during development of Dictyostelium. Differentiation 70:23-35.

47. Coates JC, Harwood AJ (2001) Cell-cell adhesion and signal transduction during Dictyostelium development. J Cell Sci 114:4349-4358.

48. Fey P, Stephens S, Titus MA, Chisholm RL (2002) SadA, a novel adhesion receptor in Dictyostelium. J Cell Biol 159:1109-1119.

49. Cornillon $\mathrm{S}$, et al. (2000) Phg1p is a nine-transmembrane protein superfamily member involved in dictyostelium adhesion and phagocytosis. J Biol Chem 275:34287-34292.

50. Bukharova T, et al. (2005) Paxillin is required for cell-substrate adhesion, cell sorting and slug migration during Dictyostelium development. J Cell Sci 118:4295-4310.

51. Nagasaki A, Kanada M, Uyeda TQ (2009) Cell adhesion molecules regulate contractile ring-independent cytokinesis in Dictyostelium discoideum. Cell Res 19:236-246.

52. Tsujioka M, et al. (2012) Talin couples the actomyosin cortex to the plasma membrane during rear retraction and cytokinesis. Proc Natl Acad Sci USA 109:12992-12997.

53. Loomis WF, Fuller D, Gutierrez E, Groisman A, Rappel WJ (2012) Innate non-specific cell substratum adhesion. PLoS One 7:e42033.

54. McCann CP, Rericha EC, Wang C, Losert W, Parent CA (2014) Dictyostelium cells migrate similarly on surfaces of varying chemical composition. PLoS One 9:e87981.

55. Tarantola M, et al. (2014) Cell substratum adhesion during early development of Dictyostelium discoideum. PLoS One 9:e106574.

56. Dias M, et al. (2016) Role of SpdA in cell spreading and phagocytosis in Dictyostelium. PLoS One 11:e0160376.

57. Vancompernolle K, Vandekerckhove J, Bubb MR, Korn ED (1991) The interfaces of actin and Acanthamoeba actobindin. Identification of a new actin-binding motif J Biol Chem 266:15427-15431.
58. Bubb MR, Korn ED (1991) Purification of actobindin from Acanthamoeba castellanii Methods Enzymol 196:119-125.

59. Lambooy PK, Korn ED (1986) Purification and characterization of actobindin, a new actin monomer-binding protein from Acanthamoeba castellanii. J Biol Chem 261:17150-17155.

60. Hertzog M, Yarmola EG, Didry D, Bubb MR, Carlier MF (2002) Control of actin dynamics by proteins made of beta-thymosin repeats: The actobindin family. I Biol Chem 277:14786-14792.

61. Bubb MR, Knutson JR, Porter DK, Korn ED (1994) Actobindin induces the accumulation of actin dimers that neither nucleate polymerization nor self-associate. $J$ Biol Chem 269:25592-25597.

62. Dan I, Watanabe NM, Kusumi A (2001) The Ste20 group kinases as regulators of MAP kinase cascades. Trends Cell Biol 11:220-230.

63. Fankhauser C, Simanis V (1994) The cdc7 protein kinase is a dosage dependent regulator of septum formation in fission yeast. EMBO J 13:3011-3019.

64. Boehme SA, Franz-Bacon K, DiTirro DN, Ly TW, Bacon KB (2016) MAP3K19 is a nove regulator of TGF- $\beta$ signaling that impacts bleomycin-induced lung injury and pulmonary fibrosis. PLoS One 11:e0154874.

65. Corbett MA, et al. (2015) A novel X-linked trichothiodystrophy associated with a nonsense mutation in RNF113A. J Med Genet 52:269-274.

66. Pellagatti A, et al. (2004) Gene expression profiling in the myelodysplastic syndromes using CDNA microarray technology. Br J Haematol 125:576-583.

67. Giorgianni F, Zhao Y, Desiderio DM, Beranova-Giorgianni S (2007) Toward a global characterization of the phosphoproteome in prostate cancer cells: Identification of phosphoproteins in the LNCaP cell line. Electrophoresis 28:2027-2034.

68. Goldfeder MB, Oliveira CC (2008) Cwc24p, a novel Saccharomyces cerevisiae nuclear ring finger protein, affects pre-snoRNA U3 splicing. J Biol Chem 283:2644-2653.

69. Lee H, Alpi AF, Park MS, Rose A, Koo HS (2013) C. elegans ring finger protein RNF113 is involved in interstrand DNA crosslink repair and interacts with a RAD51C homolog. PLoS One 8:e60071.

70. Haerty W, Artieri C, Khezri N, Singh RS, Gupta BP (2008) Comparative analysis of function and interaction of transcription factors in nematodes: Extensive conservation of orthology coupled to rapid sequence evolution. BMC Genomics 9:399.

71. Poole RJ, Bashllari E, Cochella L, Flowers EB, Hobert O (2011) A genome-wide RNAi screen for factors involved in neuronal specification in Caenorhabditis elegans. PLoS Genet 7:e1002109.

72. Carney TD, Struck AJ, Doe CQ (2013) midlife crisis encodes a conserved zinc-finger protein required to maintain neuronal differentiation in Drosophila. Development 140:4155-4164.

73. Amsterdam A, et al. (2004) Identification of 315 genes essential for early zebrafish development. Proc Natl Acad Sci USA 101:12792-12797.

74. Coene ED, et al. (2011) A novel role for BRCA1 in regulating breast cancer cell spreading and motility. J Cell Biol 192:497-512.

75. Kearse M, et al. (2012) Geneious Basic: An integrated and extendable desktop software platform for the organization and analysis of sequence data. Bioinformatics 28:1647-1649.

76. Midwood K, Orend G (2015) Special issue of Cell Adhesion \& Migration on Tenascins: Defining their role in tissue homeostasis and cancer. Cell Adhes Migr 9:1-3.

77. Li JP, Fu YN, Chen YR, Tan TH (2010) JNK pathway-associated phosphatase dephosphorylates focal adhesion kinase and suppresses cell migration. J Biol Chem 285:5472-5478.

78. Tang JP, et al. (2010) VHZ is a novel centrosomal phosphatase associated with cell growth and human primary cancers. Mol Cancer 9:128.

79. Sugiyama T, Pramanik MK, Yumura S (2015) Microtubule-mediated inositol lipid signaling plays critical roles in regulation of blebbing. PLoS One 10:e137032.

80. Plak K, Keizer-Gunnink I, van Haastert PJ, Kortholt A (2014) Rap1-dependent pathways coordinate cytokinesis in Dictyostelium. Mol Biol Cell 25:4195-4204.

81. Zhang J, Guo WH, Wang YL (2014) Microtubules stabilize cell polarity by localizing rear signals. Proc Natl Acad Sci USA 111:16383-16388.

82. Caers J, et al. (2010) Thymosin $\beta 4$ has tumor suppressive effects and its decreased expression results in poor prognosis and decreased survival in multiple myeloma. Haematologica 95:163-167.

83. Wang WS, et al. (2004) Overexpression of the thymosin beta-4 gene is associated with increased invasion of SW480 colon carcinoma cells and the distant metastasis of human colorectal carcinoma. Oncogene 23:6666-6671.

84. Ma $Y$, Yang $Y$, Wang F, Wei Q, Qin H (2015) Hippo-YAP signaling pathway: A new paradigm for cancer therapy. Int J Cancer 137:2275-2286.

85. Dou Q, et al. (2016) Ivermectin induces cytostatic autophagy by blocking the PAK1/ Akt axis in breast cancer. Cancer Res 76:4457-4469.

86. Oskarsson T (2013) Extracellular matrix components in breast cancer progression and metastasis. Breast 22:S66-S72.

87. Spenlé $C$, et al. (2015) Tenascin-C: Exploitation and collateral damage in cancer management. Cell Adhes Migr 9:141-153.

88. Boulding T, et al. (2016) Differential roles for DUSP family members in epithelial-tomesenchymal transition and cancer stem cell regulation in breast cancer. PLoS One 11 e0148065.

89. $\mathrm{Yu} \mathrm{D}$, et al. (2015) Decreased expression of dual specificity phosphatase 22 in colorectal cancer and its potential prognostic relevance for stage IV CRC patients. Tumour Biol 36:8531-8535.

90. Jeffrey KL, Camps M, Rommel C, Mackay CR (2007) Targeting dual-specificity phosphatases: Manipulating MAP kinase signalling and immune responses. Nat Rev Drug Discov 6:391-403.

91. Sussman M (1987) Cultivation and synchronous morphogenesis of Dictyostelium under controlled experimental conditions. Methods Cell Biol 28:9-29. 